\title{
How are manual skills to reach excellence in microsurgery and endovascular technique best acquired, maintained, and developed with relation to unruptured aneurysm treatment: hybrid neurosurgeons or team approach?
}

\author{
Tommy Andersson ${ }^{1,2}$ (D) \\ Received: 19 January 2021 / Accepted: 27 January 2021 / Published online: 3 February 2021 \\ (C) The Author(s), under exclusive licence to Springer-Verlag GmbH, AT part of Springer Nature 2021
}

The authors describe in the article "Microsurgical and endovascular treatment of un-ruptured cerebral aneurysms by European hybrid Neurosurgeons to balance surgical skills and medical staff Management" the experience of the socalled hybrid neurosurgeons, i.e., performing both open microvascular and endovascular operations (EVT), in the treatment of unruptured aneurysms. They find both their open surgical and endovascular results comparable to those published in the literature and argue for more such hybrid neurosurgeons to perform endovascular aneurysm treatments in Europe. I do not think that this article should be read as a comparison between techniques; there are clearly an inclusion bias, e.g., more MCA-aneurysms were treated with microsurgery whereas large and giant aneurysms were treated endovascularly, and if you claim to be equally good as somebody else, you should compare yourself with someone doing the same procedure, i.e., the literature, not with yourself doing another type of procedure. Instead, this article highlights alternative ways to organize the treatment of intracranial aneurysms, while I, having a neurosurgical background myself, sympathize with many of the statements made in the article, I think some points remain for discussion.

Knowledge, training, and experience I indeed think that neurosurgeons, just like neuroradiologists and also neurologists, should have the possibility to be trained and certified to perform EVT, and there are surely many advantages in coming from a clinical specialty when treating patients. The key for

Tommy Andersson

tommy.andersson99@gmail.com; tommy.andersson@sll.se;

tommy.andersson@azgroeninge.be

Karolinska Univ Hospital, Stockholm, Sweden

2 AZ Groeninge, Kortrijk, Belgium me is not the background of the individual but the knowledge and training that is mandatory to start achieving the experience. This has been established in several consensus documents and publications and the senior author of this article is certainly one of the pioneers in this respect [1-3]. The documents say that if you start out from one of the neuro-specialties, you still need 2 years of extra training to perform endovascular operations independently, a training tailored depending on your background. I think this makes sense; you cannot expect to be able to safely perform EVT only because you are a skilled neurosurgeon, nor is it possible for that matter if you are a skilled endovascular cardiologist with "catheter-experience." I am confident that the authors of this article certainly fulfilled all the criteria but I still think it is important to point-out the need for proper knowledge and training before starting to independently operating patients with EVT. One may then continue to gain experience, ideally in close cooperation with more experienced colleagues still supervising when needed, and experience counts, I, for instance, think that the improved results in this article when comparing EVT patients treated before and after the introduction of intracranial stents and flow-diverters, are due not only to better material and techniques but also to more experience among the operators.

Less patients for microsurgical aneurysm treatment With the rapid development of endovascular treatment for aneurysms, I agree that a problem emerges regarding training and experience for treatment by open surgery. Unless every aneurysm can be treated with EVT, there remains a need for vascular neurosurgery. The way I see a potential solution for this is to have these patients treated by fewer operators and perhaps train neurosurgeons that do similar operations and dissections for other pathologies to also manage the vascular cases. In my mind, I would think that skull-base neurosurgeons most likely 
are the most suitable in this respect, but having hybrid neurosurgeons, as described in this paper, should not per se increase the number of aneurysm patients treated surgically. A hybrid neurosurgeon should of course come to the same decision regarding treatment modality as would be the case in a team approach. Modality should be chosen based on what is believed to be best for the particular patient, not on personal preferences, ego, or availability. The choice of treatment should be independent of who makes it, so I cannot see how a hybrid neurosurgeon should make other choices than a multidisciplinary team, and it should, if done correctly, of course not lead to more patients being treated with open microsurgery. The number of aneurysms treated with open microsurgery should ideally be the same regardless of context.

Optimizing experience I disagree with the authors when they write that doing EVT would help to keep the experience and skills for open microsurgery, "to maintain neurovascular skills." I do not think that doing EVT helps you to develop and maintain surgical skills besides perhaps a better understanding of angiographical images. For instance, a hybrid neurosurgeon that for a long time only performs EVT does obviously not maintain the microsurgical skills. I think that acquiring, maintaining, and hopefully developing manual skills are very much depending on exposure and in this context; it becomes crucial to perform as many operations as possible within each subspeciality. This is obviously true for both microsurgery and EVT and you do not automatically get trained in one by doing the other. Let us, for instance, imagine that there is a center that treats 400 unruptured aneurysms per year having four physicians available. Let us assume that for practical reasons (holidays, sickness, being on-call, etc.), it is only feasible that each operator treats 100 aneurysm per year and that the best choice of treatment for the 400 patients happens to be $50 \%$ for microsurgery and EVT, respectively. In a hybrid situation, each of the four operators would then treat approximately 50 aneurysms by microsurgery and 50 by EVT to reach their 100 cases. In a team approach, with two operators doing microsurgery and two doing EVT, each of them would still treat around 100 patients but now within his or her own sub-specialty, microsurgery, or EVT. Would not the team approach then be better to achieve training and experience for both microsurgery and EVT, hopefully achieving excellence in both modalities in the institution but at different hands? In both examples, hybrid vs team, you need four people so there is no need to hire extra operators. I do realize that in the team approach, both operators have to be available, which I agree may lead to extra costs and be a practical problem with a limited number of operators at hand, but this refers more to ruptured aneurysms as you in the hybrid scenario probably need less people to cover the on-calls. In any case, this is probably the only advantage I can see for a hybrid system compared with a team approach and please note that this has nothing to do with the department that the EVT-operator belongs to. Even if all aneurysm treatments were performed by neurosurgeons belonging to a neurosurgical department, it may still be wise for the same reason to separate microsurgical treatment from EVT, so for training and experience purposes, I think the team approach may actually be superior as it allows one operator to do more cases within one subspecialty, microsurgery, or EVT. More patients to treat are always better in relation to improving skills and I do not think there is a clear cross-fertilization between open microsurgery and EVT in this respect. If you want to be a true expert in any field, I think an important advice is to treat as many patients as is practically possible.

\section{References}

1. Picard L et al (2009) Interventional neuroradiology training charter. Interv. Neuroradiol. 15(1):11-15

2. Flodmark O, Grisold W, Richling B et al (2012) Training of future interventional neuroradiologists: the European approach. Stroke. 43(10):2810-2813. https://doi.org/10.1161/STROKEAHA.112. 657882

3. European Union of Medical Specialists. UEMS recommendations for acquiring "Particular qualification" in Endovascular Interventional Neuroradiology - INR. EJMINT. 2012: 1212000052.

Publisher's note Springer Nature remains neutral with regard to jurisdictional claims in published maps and institutional affiliations. 